\title{
Editorial: A Message from the Editor-in-Chief
}

Dear friends and colleagues,

Happy New Year to you and to eNeuro.

Last year, when I was appointed as the first editor-in-chief of eNeuro, I was both excited and scared. I was excited to be given this opportunity to build a new model for scholarly publishing, but I was scared because it is a big responsibility. My chief motivation was and will always be to provide the neuroscience community with the most effective and powerful tool to publish open access online research.

At the core of eNeuro is our commitment to provide authors with a fair assessment of their work while ensuring that we publish only excellent science. In order to do that we need to meet three requirements: authors must be able to identify respected scientists in the field to serve as Reviewing Editors, we must deliver a fair reviewing process, and we must provide a clear decision.

Here's how eNeuro is meeting those requirements:

1. eNeuro has an extensive panel of 58 Reviewing Editors (which will expand), all active top scientists, with an equal gender (50-50) and geographical (50 percent non-US-based) distribution.

2. We have established a double-blind system. Wherever possible reviewers cannot identify the authors when reading the manuscript.

3. The Reviewing Editor and the reviewers consult each other to send a consensus message and decision to the authors. There cannot be two opposite reviews conveyed to the authors. If an agreement cannot be reached with two opposing reviewers, a third reviewer is engaged.

Does it work? Given the unsolicited praise we received from authors and reviewers, we believe that we have achieved our goal in terms of fairness. Of course, no system is perfect, but the goal is to make it as satisfying to everyone as it can be.

I am thrilled to announce that eNeuro has published more than 100 papers in its first year of existence. This is more than any other commercial and non-commercial open access journal publishing neuroscience papers.

The community's trust in the Society for Neuroscience is particularly rewarding, all the more since we were indexed in PubMed this October after a year-long wait.

Of course, a recurrent theme is that of the impact factor. Authors always ask us: "Should I send my science to a journal that does not have an impact factor?" It takes two years to receive one. But given the high quality of the papers we have already published, and their number, you can be confident that eNeuro should be on top of your list of journals.

Finally, I would like to emphasize once more that eNeuro strives to serve our community. This means that we are not only publishing excellent, cutting-edge science. We are also publishing negative results (thus preventing other groups from testing the same hypotheses), failures to reproduce (there are many irreproducible published results that we should be aware of), and replication results (adding weight to major theories). In addition, eNeuro publishes reviews and method papers as well as commentaries and opinions to foster discussion.

This has been a very exciting and fulfilling first year. Thank you for trusting eNeuro, and we hope to see you soon.

\section{Christophe Bernard}

Editor-in-Chief

DOI:10.1523/ENEURO.0162-15.2016

\section{Note from the Publisher}

SfN's two journals, JNeurosci and eNeuro, reflect a commitment to the excellence, rigor, and breadth of scientific publishing for which the Society for Neuroscience is known. To reflect and serve an exciting and evolving field, the Society's publishing options are also evolving. SfN journals offer neuroscientists more choices for how to publish, while you can be confident you are publishing in journals with a reputation for excellence that reinvest in the long-term growth of the neuroscience field. Building on the historic strengths of JNeurosci and the innovative publishing models of eNeuro, the two journals' editors-in-chief, Marina Picciotto and Christophe Bernard, as well as their editorial boards, are exploring how they can best serve publishing needs in the field. Al- ready, they are identifying opportunities to define the studies that will be most appropriate to each journal's format.

JNeurosci will focus on in-depth, well-rounded studies of high scientific quality and impact. eNeuro will have a similar breadth and focus on scientific soundness, but it will also publish studies that present new observations that may not yet have mechanistic underpinnings, as well as papers describing new tools, theories or concepts, negative results, failure to replicate, and confirmations. In keeping with this focus, eNeuro will consider Brief Communications based on the same criteria as any other paper. This is a natural development because manuscripts that reviewers may now consider too preliminary for JNeurosci could be highly 
appropriate for eNeuro. JNeurosci will still publish short manuscripts and have no lower or upper limit on the number of figures; it will focus on well-rounded papers regardless of length.

To support the complementary roles of JNeurosci and eNeuro, an efficient manuscript transfer process for authors who elect to move scientifically sound papers between the two journals has been developed by the editorial boards of the two journals. If they are not accepted in the journal originally selected by the authors, these articles will be considered by the sister journal, sometimes without additional review. With this interactive relationship, the journals will provide new options for rapid publication, all handled and reviewed by working scientists who have been nominated based on their service to the journals through frequent, thoughtful and constructive manuscript reviews. The addition of eNeuro will allow SfN to publish more articles based on sound science, including those that reviewers might judge to have less depth or a different impact than those required for publication in JNeurosci. As a result, we expect more opportunities for our authors to get their manuscripts published in a journal published by SfN.

The Society thanks authors and members for your strong support. We welcome your ongoing feedback on what we are doing well, and what we can do better. We look forward to serving you and encourage you to submit your excellent science to The Journal of Neuroscience and eNeuro. 